\title{
Recognition of Physiotherapeutic Exercises through DTW and Low-Cost Vision-Based Motion Capture
}

\author{
Yves Rybarczyk $^{1,2, *}$, Jan Kleine Deters ${ }^{3}$, Arián Aladro Gonzalo ${ }^{1}$, Danilo Esparza ${ }^{1,4}$, \\ Mario Gonzalez ${ }^{1}$, Santiago Villarreal ${ }^{1}$, Isabel L. Nunes ${ }^{2}$ \\ ${ }^{1}$ Universidad de Las Americas, Intelligent \& Interactive Systems Lab ( $\left.\mathrm{SI}^{2} \mathrm{Lab}\right) \mid$ Research \\ Laboratory in Physiotherapy and Human Movement, Av. de los Granados E12-41 y Colimes \\ esq., Quito - EC170125, Ecuador \\ \{yves.rybarczyk, arian.aladro, mario.gonzalez, santiago.villarreal, \\ danilo.esparza\}@udla.edu.ec \\ ${ }^{2}$ Nova University of Lisbon, Department of Electrical Engineering, UNINOVA/CTS | De- \\ partment of Mechanical \& Industrial Engineering, UNIDEMI, Monte de Caparica, Portugal \\ $\{$ y.rybarczyk, imn\}@fct.unl.pt \\ ${ }^{3}$ University of Twente, Drienerlolaan 5, 7522 NB Enschede, The Netherlands \\ j.kleinedeters@student.utwente.nl \\ ${ }^{4}$ Pontificia Universidad Católica del Ecuador, Quito, Ecuador
}

\begin{abstract}
Telemedicine is a current trend in healthcare. The present study is part of the ePHoRt project, which is a web-based platform for the rehabilitation of patients after hip replacement surgery. To be economically suitable the system is intended to be based on low-cost technologies, especially in terms of motion capture. This is the reason why the Kinect-based motion tracking is chosen. The paper focuses on the automatic assessment of the correctness of the exercises performed by the user. A Dynamic Time Warping (DTW) approach is used to discriminate between correct and incorrect movements. The classification of the movements through a Naïve Bayes classifier shows a very high percentage of accuracy (98.2\%). Models are built for each individual and reeducation exercise with only few attributes and the same accuracy. Due to these promising results, the next step will consist of testing the algorithms on patients performing the exercises in real time.
\end{abstract}

Keywords: Telerehabilitation, movement assessment, Dynamic Time Warping, machine learning, Kinect-based motion tracking.

\section{Introduction}

A current trend in medicine is home therapy systems. This concept consists of enabling patients to carry out part of the rehabilitation at home and to communicate through the Web the evolution of the recovery process. Thus, health professionals can proceed with a remote monitoring of the patient's performance and an adaptation of the treatment accordingly. In Ecuador, telerehabilitation systems are still little devel-

${ }^{*}$ Corresponding author 
oped. However, this technology could bring several advantages for the individual and the society in terms of healthcare (improvement of the recovery process by the possibility to perform rehabilitation exercises more frequently), economy (reduction of the number of medical appointments and the time patients spend at the hospital), mobility (diminution of the transportation to and from the hospital) and ethics (healthcare democratization and increased empowerment of the patient).

By taking into account these considerations, the project ePHoRt proposes to develop a Web-based platform for home motor rehabilitation. The tool is developed for patients after hip arthroplasty surgery. This orthopedic procedure is an excellent case study, because it involves people who need a postoperative functional rehabilitation program to recover strength, function and joint stability. In addition, due to the condition of the patients, it is difficult to carry them to the hospital. The project intends to tackle three main issues. First, the motion capture technology must be low-cost, in order to be used worldwide. Second, the system should automatically detect the correctness of the executed movement, in order to provide the patient with real time feedback. Third, new computational approaches have to be researched, in order to promote patient's motivation to regularly complete the rehabilitation tasks (e.g., by the use of affective computing and serious games paradigms to detect difficulties and stimulate effort in the patients, respectively).

This paper focuses on the two first parts of the project, which are the development of a program to automatically assess the correctness of the movements by using an information provided by a low-cost motion capture device. The manuscript is divided into five sections. The first part is a state of the art in terms of technologies for home rehabilitation and telemedicine. The second is a description of the architecture that will support the platform. The third exposes the methodology used to evaluate the movement. The fourth part consists of a presentation of the results. Finally, some conclusions and perspectives are drawn up regarding the midterm progress of the project.

\subsection{State of the Art}

In any kind of rehabilitation, repeated exercises of an impaired limb maximize the chances of recovery [1]. In practice, medical and economic situations limit the number of therapeutic sessions the patient can take at the hospital or medical centers. This fact justifies the increased trend in developing telehealthcare systems to enhance home therapy. However, this new approach of healthcare copes with several other obstacles. First, the used technology must be both reliable and affordable in order to significantly reduce the costs. Second, the system has to provide a rigorous and real time monitoring of the patient's movements to make sure that the rehabilitation protocol is properly executed. For instance, it has to detect the patients' tendency to compensate their diminished limb with other functional part of their body, which makes rehabilitation progress slower [2]. Third, providing a therapeutic framework at home does not automatically implicate a speedy recovery, because of the lack of motivation among patients to exercise for sustainable period of time.

Home-based rehabilitation has gained prominence over the recent years through the development of exercise platform [3], virtual reality-based system [4], gaming console [5] and the widely popular Kinect camera-based system [6]. For the purpose 
of this project it is necessary to make a survey on three critical points regarding home therapy. First, the existing technologies used in telemedicine for patient's rehabilitation. Second, systems and methods developed to perform a real time analysis and recognition of the movement. Third, the main approaches implemented to enhance motivation in patients to regularly complete the exercises. In the context of this paper that focuses on the motion capture and assessment parts of the project, the state of the art is based on the first two points, only.

Motion Capture Systems. Motion capture is used in a wide range of areas, from the entertainment industry (e.g., three-dimensional animation) [7] to scientific studies, such as biological motion analysis [8]. However, it can be an expensive and complex technology that it is not always usable at home. For instance, one of the most famous motion capture systems for professional use is the Vicon motion system. This kind of technology is based on light-reflecting markers positioned on the individual body and infrared-camera sensors that enable a precise analysis of movements. The main limitations of this tool are its high-cost and the fact that it only works in very controlled conditions (usually in laboratories). A quite more affordable equipment is the popular Kinect camera from Microsoft. This system has several advantages over the other technologies. It is a cheap motion capture device (around 100USD). It is quite easy to install and use at home. And it provides an automatic reconstruction of the threedimensional coordinates of the body main joints, with a reasonable spatial and temporal resolution [9]. A study comparing the Vicon and the Kinect system for measuring movement in people with Parkinson's disease shows that the Kinect has the potential to be a suitable equipment to capture gross spatial characteristics of clinical relevant movements [10]. Also, a computer vision approach based on the use of CCD webcams can be implemented, but the apparatus usually requires two optical sensors in order to calculate the 3D-coordinates by triangulation and it needs the development of additional image processing algorithms [11]. An attempt to reconstruct body postures and 3D movements from monocular video sequences was proposed by [12], but the true accuracy and applicability for real-time tele-rehabilitation are not yet demonstrated. Finally, an alternative to vision-based monitoring is a wearable system. However, the use of complicated wearable devices makes it tiresome and, consequently, tends to decrease the effectiveness of rehabilitation exercises [13]. A preliminary experiment for the ePHoRt project shows a significant correlation between the accuracy of a Kinect and an accelerometer sensor, which demonstrates that a vision-based motion capture is an excellent alternative to an inertial-based motion capture [14].

Movement Recognition and Assessment. Human action recognition is a very challenging topic. It presents more degrees of freedom with respect to system design and implementation when compared to language processing [15]. Different approaches are used regarding the sensor technologies and computational algorithms. The first kind of sensors are wearable devices, which are based on pervasive and mobile computing $[16,17]$. A less invasive technique consists of using a vision-based recognition. The Kinect camera has largely contributed to the growth of this approach, due to the fact that it facilitates the extraction of the pertinent features for gesture recognition [18] [19]. Movement recognition has to be carried out through a process flow that usually 
involves raw data recording and filtering, feature extraction and selection, and classification by the use of machine learning models. Techniques of time series analysis are applied to compare the similarity between two temporal sequences, such as Dynamic Time Warping [20]. A wide range of classifiers have been used for action recognition in the last decades [21]. One of the most successful methods to achieve recognition of daily activity is the discriminative approach. This classification is based on the construction of decision boundaries in the feature space, specifying regions for each class. The main classifiers that implement this type of method for activity recognition are the k-Nearest Neighbour [22], Support Vector Machines [23], the Naïve Bayes [24], and C4.5 Decision Tree [25]. The last two are by far the most popular algorithms, because they generally enable a high classification accuracy [26] and the hierarchical tree representation makes the model easier to read than a sequence of rules [27].

\subsection{Platform Architecture}

The ePHoRt project is a Web application based on a three layers Client-Server architecture (Fig. 1). The client layer (browser) will be developed in JavaScript by the use of two possible frameworks: jQuery or AngularJS. The application layer (domain server) will be implemented either in Django framework from Python or Java framework. The data server layer (database) will be developed in MySQL.

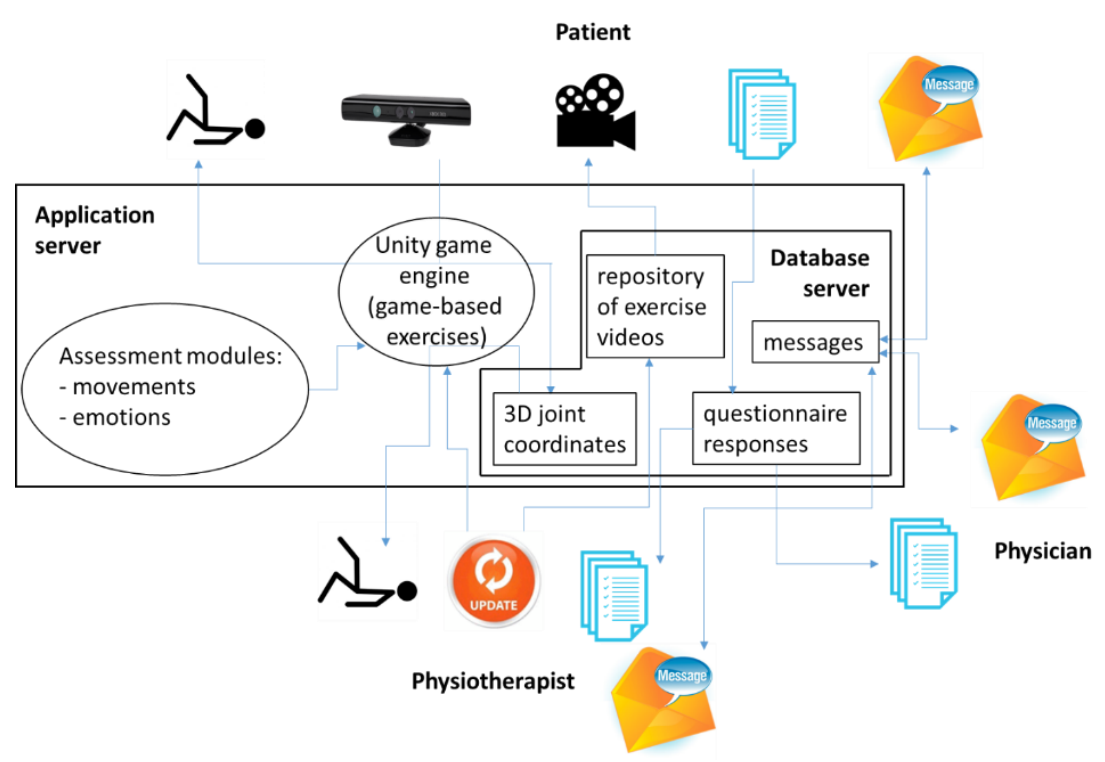

Fig. 1. ePHoRt platform architecture.

A different user interface will be implemented for each of the three kinds of user: patient, physiotherapist and physician. After login, the patients will have an access to the programmed exercises they have to achieve. The patient's interface will be composed with two dynamic frames: (i) one to display a video example of the exercises to 
be completed and (ii) another to display a 3D-avatar that will mimic in real-time the patient's movements captured by the Kinect. The patient will receive a real-time feedback regarding the correctness of the movements, thanks to the assessment module, and game scores. In addition, questionnaires will be available on this interface, in order to record qualitative information. The main functionalities of the physiotherapist's interface will be: (i) accessing to the patient's performance for each exercise, (ii) consulting the answers to the self-reported functions, (iii) watching the threedimensional reproduction of the patient's movement through an animated avatar and (iv) updating (to more challenging parameters of the same exercise or to new kinds of exercise) the rehabilitation program according to the patient's progress and/or medical advices. Finally, an interface for the physicians will enable them to supervise all the recovery process and communicate with the physiotherapist to authorize or not certain movements according to the specific condition of each patient. The communication between the three stakeholders will be supported by an exchange of messages.

The application layer will contain the logical structure of the platform. This intermediate layer will be connected to the client, through Internet, and to the database server. It will receive and process the requests from the three types of user. Here, the Unity 3D game engine will be used to develop the game-based exercises and animate the avatar. Also, the affective and movement assessment modules will be integrated at this level.

The database layer will be connected to the application layer. Heterogeneous data will be stored into the database, such as: (i) quantitative data about the 3D-coordinates of the movements, (ii) qualitative data about the responses to the questionnaires, (iii) videos of the exercises and (iv) comments made by the physiotherapists and physicians.

\section{Automatic Assessment of the Correctness of the Movement}

\subsection{Material and Method}

Experimental Protocol. Eight subjects participated in the experiment. They were asked to execute four different rehabilitation movements. Each movement was repeated eleven times: six times correctly and five times imperfectly. The correctness of the exercises was labeled by a physiotherapist. The first movement was a hip abduction (HA). The second movement was a hip extension (HE). The third movement was a slow flexion of hip and knee (SFHK). And the last movement was a sequence, in which the subject had to do one step forward, one step sideways and one step backward (FSB). All of these movements were performed on the right side, only. For these rehabilitation exercises the main mistakes that an individual can do are: (i) an inappropriate amplitude of the movement (too short or too large), (ii) an additional flexion of joints not involved in the exercise (e.g., trunk flexion or extension), (iii) an execution of the movement in the wrong spatial plane, (iv) an incorrect positioning of the center of mass. All of these errors were used as imperfect trials of the experiment. During the execution of the movements, subjects were in stand up position and at approximatively 2.5 meters from a Kinect camera. The Kinect height was aligned 
with the xiphoid apophysis of the subjects. A program was developed to record the 3D-coordinates $(\mathrm{X}, \mathrm{Y}, \mathrm{Z})$ of each joint of the Kinect Skeleton. Thus, twenty joints were analyzed. The framerate of the motion capture was $33 \mathrm{~Hz}$, approximately.

Time Series Analysis. A Dynamic Time Warping (DTW) analysis was used to assess the correctness of the movement. This method measures the similarity between two temporal sequences which may vary in speed [28]. Thus, similarities in rehabilitation exercises can be detected, even if the referential movement (the movement correctly executed by the patient) is not executed at exactly the same velocity when performed at home. This technique is based on the calculation of the distance between all pair of points in two signals (distance $(\mathrm{i}, \mathrm{j})$ ). Lesser distance implies that these points may be candidates to be matched together. In order to find the minimum distance, an accumulated cost matrix (D) is calculated as follows:

$$
\mathrm{D}(\mathrm{i}, \mathrm{j})=\min \{\mathrm{D}(\mathrm{i}-1, \mathrm{j}-1), \mathrm{D}(\mathrm{i}-1, \mathrm{j}), \mathrm{D}(\mathrm{i}, \mathrm{j}-1)\}+\operatorname{distance}(\mathrm{i}, \mathrm{j})
$$

For movement assessment, the signal of the movement to be assessed is compared to the signal of the movement correctly executed (correct trials). In the present protocol six trials per exercises were evaluated as correct by the therapist. Thus, the DTW algorithm was applied by the use of six different referential signals. At the end, fortyfive distances were calculated for each of the three axes of the whole joints. Thirty of these observations were labeled as incorrect movements and fifteen as correct movements. Then, these data were used as input of a machine learning algorithm of classification, in order to evaluate if the DTW is an accurate technique to discriminate the correct from the incorrect execution of the movements.

Machine Learning Algorithm for Classification. The assessment of the DTW approach is carried out through a machine learning classification. If a high percentage of correct classification is obtained, it will mean that the DTW is an appropriate technique to differentiate the right from the wrong movements. As described in the introduction, several classifiers are possible. Here, the Naïve Bayes classifier is chosen because we make the assumption that all of the features (coordinates X,Y,Z of each joint) contribute equally and independently to the decision [29]. This method is based on the "Bayes Theorem" of the probability (Pr) of an event $\mathrm{H}$ (class of an instance) given an evidence $\mathrm{E}$ (attribute values of the instance), such as:

$$
\operatorname{Pr}(\mathrm{H} \mid \mathrm{E})=\operatorname{Pr}(\mathrm{E} \mid \mathrm{H}) \operatorname{Pr}(\mathrm{H}) / \operatorname{Pr}(\mathrm{E})
$$

$\operatorname{Pr}(\mathrm{H})$ is called the priori, or baseline, probability of the hypothesis $\mathrm{H}$. That is the probability of the event before any evidence is seen. $\operatorname{Pr}(\mathrm{H} \mid \mathrm{E})$ is a posteriori probability of $\mathrm{H}$, after an evidence is seen (E). The naïve assumption is that the evidence splits into parts that are statistically independent. The parts of the evidence in the movement assessment are the sixty coordinates $(20 \times 3)$ in the joints motion dataset. When the events are independent the probabilities multiply, such as:

$$
\operatorname{Pr}(H \mid E)=\operatorname{Pr}\left(E_{1} \mid H\right) \operatorname{Pr}\left(E_{2} \mid H\right) \ldots \operatorname{Pr}\left(E_{60} \mid H\right) \operatorname{Pr}(H) / \operatorname{Pr}(E)
$$


There are two hypotheses $(\mathrm{H})$ for the movement assessment: correct vs. incorrect. To get a probabilistic value (between 0 and 1), the likelihood of each hypothesis (or class) is normalized. The main possible issue in using Naiive Bayes method is in case of redundant attributes. In this situation it is possible to use additional methods for feature selection, in order to select a subset of fairly independent attributes.

Feature Selection. Not all of the sixty attributes are essential to proceed with the assessment of the movement. Thus, a selection of the relevant attributes for each kind of exercises could be performed, in order to improve the classification (correct vs. incorrect) of the movement (elimination of redundant features) and to get a simplified model of assessment of the correctness of the movement (only based on the most pertinent features). Several techniques are available to automatically perform this selection. The method used in this study is the "wrapper" attribute selection [30]. This method can be applied backward, forward or bi-directional. The backward searching consists of removing one attribute (the worst one) at each search step. On the contrary, the forward searching start with a zero attribute subset and add the best attribute each time. The bi-directional is a combination of backward and forward searching. In all of the cases, the search stops when the classification performance gets worse. The "wrapper" method uses cross-validation to select the best attribute to add or to drop at each stage. To sum up, two components have to be defined to apply this technique in practice: a search method and an attribute evaluator. The search method defines the searching direction and the search termination criteria. The attribute evaluator evaluates feature sets by using a learning scheme and classifier. In this study, the setup used is backward searching and Naïve Bayes classifier.

\subsection{Results}

Experimental Results. Overall, the average classification accuracy of the movements is $98.2 \%(\mathrm{SD}=1.1)$. The mean of accurate assessments for each movement is higher than $97 \%$. Table 1 shows that the accuracy of this classification is almost the same between the different exercises and the different subjects. These results suggest that the DTW is an appropriate technique to discriminate between correct and incorrect execution of the rehabilitation movements.

Table 1. Percentage of accuracy in the assessment of the movements.

\begin{tabular}{c|cccc|c}
\hline Subjects & HA & FSB & HE & SFHK & Mean \\
\hline 1 & .96 & 1 & 1 & 1 & .99 \\
2 & 1 & 1 & .98 & .96 & .985 \\
3 & .91 & 1 & 1 & .93 & .96 \\
4 & .96 & 1 & .98 & .98 & .98 \\
5 & 1 & 1 & .96 & 1 & .99 \\
6 & 1 & .96 & 1 & 1 & .99 \\
7 & .98 & .93 & 1 & .96 & .968 \\
8 & 1 & 1 & .96 & 1 & .99 \\
\hline Mean & .976 & .986 & .985 & .979 & .982 \\
\hline
\end{tabular}


After feature selection the overall percentage of accurate classification of the movements is $98 \%$. As previously, none of the movements have a classification lower than $97 \%$. The similarity between the results with and without attribute selection is confirmed by a T-test analysis that shows no significant differences between the classification performance on these two datasets $(p=.7)$. It is to note that the value of the standard deviation is slightly lower when a selection of features is performed $(\mathrm{SD}=$ .71). This fact suggests that the inter-individual differences in the assessment of the movements are reduced when they are based on a selection of the most relevant attributes for each exercise and individual. Figure 2 shows a comparison of the classification accuracy, for each movement and subject, between the two datasets. Even on this detailed analysis it is possible to confirm that the quality of the assessment with the selected features is as good as with the totality of the features. This result suggest that it is preferable to build a model based on a selection of the most relevant attributes than to use the whole features, because we will get a simple model as accurate as a complex one. This characteristic will be fundamental when the model will have to assess the correctness of the movements in real time.

HA
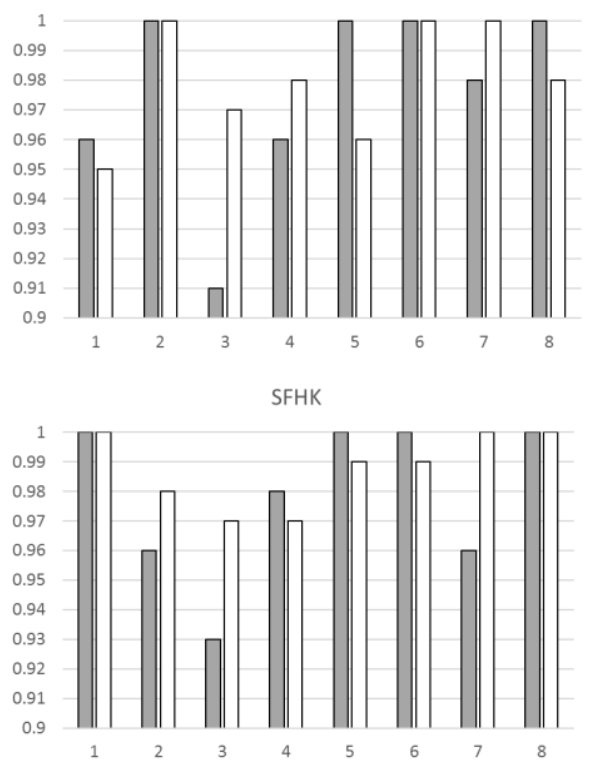

FSB

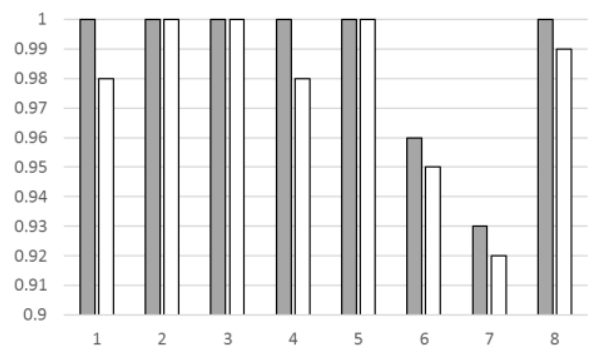

$\mathrm{HE}$

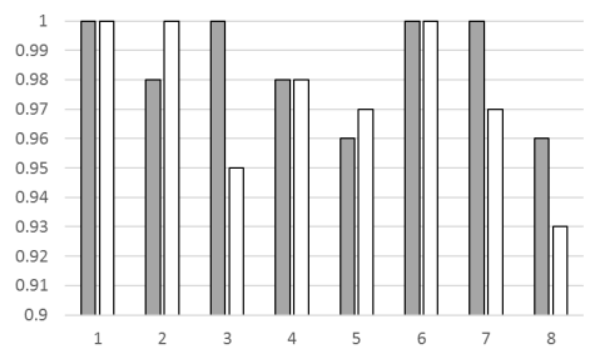

Fig. 2. Comparison of classification accuracy (in \%) between the whole feature dataset (grey bars) vs. the selected feature dataset (white bars) for each exercise and individual.

Models. Different models were created for each subject and exercise through a feature selection based on a wrapper technique. Table 2 shows a synthesis of the main joints involved in the assessment of the rehabilitation movements. The main features that enables the algorithm to discriminate between a correct and incorrect movement are related with the "Right Foot", the "Right Ankle" and the "Right Knee". This is not surprising considering that all of the exercises asked to the participants were designed 
for the rehabilitation of the "Right Hip". Taking together, these three joints represent $78 \%$ of the features used for the assessment. The other $22 \%$ are represented by different kind of joints according to the exercise and the individual. With $62.5 \%$ of "Other Joints", "Hip Abduction" is the movement with the largest inter-individual variability. On the contrary the "Forward, Sideways and Backward" sequence is the only exercise that can be exclusively assessed on the base of the lower limbs (100\%) involved in the movement (mostly foot kinematics). Also, "Hip Extension" and "Slow Flexion of Hip and Knee" are mainly evaluable through an analysis of the lower limbs in movement with $80 \%$ and $90 \%$ of the recognition based on these joints, respectively.

Table 2. Percentage of the joints used to assess each exercise.

\begin{tabular}{c|cccc}
\hline Exercises & R Foot & R Ankle & R Knee & Other Joints \\
\hline HA & .375 & 0 & 0 & .625 \\
FSB & .625 & .1 & .25 & 0 \\
HE & .4 & .2 & .2 & .2 \\
SFHK & .5 & .3 & .1 & .1 \\
\hline Mean & .48 & .15 & .15 & .22 \\
\hline
\end{tabular}

To sum up, half of the assessment of the exercises depends on the kinematic of the "Right Foot". Then, the "Right Ankle", "Right Knee" and the rest of the joints contribute almost equally for the evaluation of the correctness of the movement. However, it is difficult to get a generic model that could be used to assess anybody, because more than $20 \%$ of the features represent joints that are not directly involved in the movement. Thus, the best and easier way to assess the movement is to create a specific model for each individual and exercise. For instance, Table 3 shows the models obtained for the subject $n^{\circ} 5$.

Table 3. Models for the subject ${ }^{\circ} 5$ to assess the whole therapeutic exercises.

\begin{tabular}{c|c|cc|cc}
\hline Exercises & Attributes & \multicolumn{2}{|c}{ Incorrect } & \multicolumn{2}{c}{ Classes } \\
& & & Correct \\
& & Mean & SD & Mean & SD \\
\hline HA & Head - Y & 2.67 & 1.92 & .06 & .07 \\
& R Foot - X & 2.08 & .92 & 1.11 & .19 \\
\hline FSB & R Foot - Z & 267805 & 93179 & 63722 & 15659 \\
\hline HE & R Elbow - Z & 37712 & 24606 & 5758 & 1857 \\
& R Foot - Y & 2.57 & 1.33 & 1.39 & .29 \\
\hline SFHK & R Foot - Z & 151958 & 69724 & 29849 & 4175 \\
\hline
\end{tabular}

In the case of this individual the algorithm only needed two features to discriminate between a correct and incorrect "Hip Abduction" ("Head" in the y-axis and "Right Foot" in the X-axis). For the "FSB" movement a single attribute was sufficient for the discrimination ("Right Foot" in the z-axis). Again, only two attributes were necessary for the assessment of the "HE" exercise ("Right Elbow" in the z-axis and "Right Foot" in the y-axis). And the "SFHK" was assessed by a single attribute, as well ("Right Foot" in the z-axis). The value of the mean of each feature corresponds to the 
distance between the referential movement and the tested movement calculated by the DTW technique. Data in table 3 confirm that the mean and the standard deviation are always lower for the correct movements than the incorrect movements.

Through these models it is possible to estimate the correctness of a new movement executed by this subject, based on the calculation of the two probabilities of this movement for being correct and incorrect. The example of the "Hip Abduction" is used to explain the methodology applied to assess the movement from its model. The method is based on the use of the distribution of the numerical variable (attribute values) to have a good guess of the frequency. One common practice is to assume normal distributions for the numerical variables. The probability density function for the normal distribution is defined by two parameters: mean $(\mu)$ and $\operatorname{SD}(\sigma)$. These two values are provided by the model in table 3 for each selected feature of a determined movement. This probability is obtained through the calculation of the value of the normal distribution of an attribute value (x), by the use of the equation 4 .

$$
f(x)=\frac{1}{\sigma \sqrt{2 \pi}} e^{-\frac{(x-\mu)^{2}}{2 \sigma^{2}}}
$$

Thus, in the case of the "Hip Abduction" for subject $\mathrm{n}^{\circ} 5$, the probabilities to assess a new movement as correct and incorrect by the use of the "Head distance in the yaxis" $\left(d_{\text {headY }}\right)$ will be obtained by the calculation of the equations 5 and 6 , respectively.

$$
\begin{aligned}
& \operatorname{Pr}\left(d_{\text {headY }} \mid \text { correct }\right)=\frac{1}{0.07 \sqrt{2 \pi}} e^{-\frac{\left(d_{\text {headY }}-0.06\right)^{2}}{2(0.07)^{2}}} \\
& \operatorname{Pr}\left(d_{\text {headY }} \mid \text { incorrect }\right)=\frac{1}{1.92 \sqrt{2 \pi}} e^{-\frac{\left(d_{\text {headY }}-2.67\right)^{2}}{2(1.92)^{2}}}
\end{aligned}
$$

The same calculation has to be applied to get the probabilities with the feature "Right Foot distance in the $\mathrm{x}$-axis" $\left(\mathrm{d}_{\mathrm{rFoot}}\right)$, as described by the equations 7 and 8 .

$$
\begin{aligned}
& \operatorname{Pr}\left(d_{\text {rFootX }} \mid \text { correct }\right)=\frac{1}{0.19 \sqrt{2 \pi}} e^{-\frac{\left(d_{r F o o t X}-1.11\right)^{2}}{2(0.19)^{2}}} \\
& \operatorname{Pr}\left(d_{\text {rFootX }} \mid \text { incorrect }\right)=\frac{1}{0.92 \sqrt{2 \pi}} e^{-\frac{\left(d_{r F o o t X}-2.08\right)^{2}}{2(0.92)^{2}}}
\end{aligned}
$$

Then, the value of each probability is used to calculate the likelihood (LL) for a movement classification as correct and incorrect. The calculation of the two likelihoods is described in the equations 9 and 10 .

$$
\begin{gathered}
\mathrm{LL}(\text { correct })=\operatorname{Pr}\left(\mathrm{d}_{\text {headY }} \mid \text { correct }\right) \operatorname{Pr}\left(\mathrm{d}_{\mathrm{rFootX}} \mid \text { correct }\right) \operatorname{Pr}(\text { correct }) \\
\mathrm{LL}(\text { incorrect })=\operatorname{Pr}\left(\mathrm{d}_{\text {headY }} \mid \text { incorrect }\right) \operatorname{Pr}\left(\mathrm{d}_{\mathrm{rFootX}} \mid \text { incorrect }\right) \operatorname{Pr}(\text { incorrect })
\end{gathered}
$$

Finally, these likelihoods have to be normalized to get the value of the probabilities for the new movement to be correct and incorrect (see equations 11 and 12). 50\% is 
usually used as a threshold to take the final decision to classify the trial in one or the other class.

$$
\begin{gathered}
\operatorname{Pr}(\text { correct })=\operatorname{LL}(\text { correct }) /(\operatorname{LL}(\text { correct })+\operatorname{LL}(\text { incorrect })) \\
\operatorname{Pr}(\text { incorrect })=\operatorname{LL}(\text { incorrect }) /(\operatorname{LL}(\text { correct })+\operatorname{LL}(\text { incorrect }))
\end{gathered}
$$

\section{Conclusions and Perspectives}

The presented study is part of the ePHoRt project, which is a telerehabilitation platform for patients after hip replacement surgery. It focuses on the automatic assessment of the correctness of the exercises performed by the user. The results show that a DTW approach permits a high level of discrimination between correct and incorrect execution of the movements $(98.2 \%)$. The implementation of a feature selection technique allows us to build simple models only based on few attributes to evaluate the movement. Future work will consist of (i) a DTW analysis on the joint angles and (ii) testing the algorithms in real time with patients.

\section{References}

1. Feys, H., De Weerdt, W., Verbeke, G. et al.: Early and repetitive stimulation of the arm can substantially improve the long-term outcome after stroke: a 5-year follow-up study of a randomized trial. Stroke, 35(4), 924-929 (2004)

2. Cramp, M.C., Greenwood, R.J., Gill, M. et al.: Effectiveness of a community-based low intensity exercise programme for ambulatory stroke survivors. Disabil. Rehabil., 32(3), 239247 (2010)

3. Mavroidis, C., Nikitczuk, J., Weinberg, B. et al.: Smart portable rehabilitation devices. J. Neuroeng. Rehabil., 2, 18 doi:10.1186/1743-0003-2-18 (2005)

4. Holden, M.K., Dyar, T.A., Dayan-Cimadoro, L.: Telerehabilitation using a virtual environment improves upper extremity function in patients with stroke. IEEE Trans. Neural Syst. Rehabil. Eng., 15(1), 36-42 (2007)

5. Rand, D., Kizony, R., Weiss, P.T.L.: The Sony PlayStation II EyeToy: low-cost virtual reality for use in rehabilitation. J. Neurol. Phys. Ther., 32(4), 155-163 (2008)

6. Oikonomidis, I., Kyriazis, N., Argyros, A.A.: Efficient model-based 3D tracking of hand articulations using Kinect. In: Proceedings of the 22nd British Machine Vision Conference. University of Dundee (2011)

7. Gameiro, J., Cardoso, T., Rybarczyk, Y.: Kinect-Sign: Teaching sign language to listeners through a game. In: Rybarczyk, Y. et al. (eds.). Innovative and Creative Developments in Multimodal Interaction Systems, pp. 141-159. Springer, Heidelberg (2014)

8. Rybarczyk, Y., Santos, J.: Motion integration in direction perception of biological motion. In: Proceedings of the 4th Asian Conference on Vision. Matsue (2006)

9. Dutta, T.: Evaluation of the Kinect sensor for 3-D kinematic measurement in the workplace. Applied Ergonomics, 43, 645-649 (2012)

10.Brook, G., Barry, G., Jackson, D., Mhiripiri, D., Olivier, P., Rochester, L.: Accuracy of the Microsoft Kinect sensor for measuring movement in people with Parkinson's disease. Gait \& Posture, 39(4), 1062-1068 (2014) 
11.Rybarczyk, Y.: 3D markerless motion capture: a low cost approach. In: Proceedings of the 4th World Conference on Information Systems and Technologies. Recife (2016)

12.Remondino, F., Roditakis, A.: 3D reconstruction of human skeleton from single images or monocular video sequences. In: Proceedings of Joint Pattern Recognition Symposium. Magdeburg (2003)

13.Krukowski, A., Vogiatzaki, E., Rodríguez, J.M.: Patient health record (PHR) system. In: Maharatna K. et al. (eds.). Next generation remote healthcare: a practical system design perspective, chapter 6. Springer, New York (2013)

14.Rybarczyk, Y., Kleine Deters, J., Aladro Gonzalvo, A., Gonzalez, M., Villarreal, S., Esparza, D.: ePHoRt project: a web-based platform for home motor rehabilitation. In Proceedings of the 5th World Conference on Information Systems and Technologies. Madeira (2017)

15.Bulling, A., Blanke, U., Schiele, B.: A tutorial on human activity recognition using bodyworn inertial sensors. ACM Comput. Surv., 46(3), doi:10.1145/249962 (2014)

16.Chen, L., Hoey, J., Nugent, C.D. et al.: Sensor-based activity recognition. IEEE Trans. Syst. Man Cybern. Part C Appl. Rev., 42(6), 790-808 (2012)

17.Choudhury, T., Consolvo, S., Harrison, B. et al.: The mobile sensing platform: an embedded activity recognition system. IEEE Pervasive Comput., 7(2), 32-41 (2008)

18.Galveia, B., Cardoso, T., Santos, V., Rybarczyk, Y.: Towards the creation of a gesture library. EAI Endorsed Transactions on Creative Technologies, 15.3, e3 (2015)

19.Jost, H.: Kinect-based approach to upper limb rehabilitation. In: Vogiatzaki, E. \& Krukowski, A. (eds.). Modern Stroke Rehabilitation through e-Health- based Entertainment, pp. 169-193. Springer, Heidelberg (2016)

20.Antón, D., Goñi, A., Illarramendi, A.: Exercise recognition for Kinect-based telerehabilitation. Methods Inf. Med., 54(2), 145-155 (2015)

21.Jain, A.K., Duin, R.P.W., Mao, J.: Statistical pattern recognition: a review. IEEE Trans. Pattern Anal. Mach. Intell., 22(1), 4-37 (2000)

22.Kunze, K., Barry, M., Heinz, E.A. et al.: Towards recognizing tai chi: an initial experiment using wearable sensors. In: Proceedings of the 3rd International Forum on Applied Wearable Computing. Bremen (2006)

23.Banos, O., Damas, M., Pomares, H. et al.: Daily living activity recognition based on statistical feature quality group selection. Expert Syst. Appl., 39(9), 8013-8021 (2012)

24.Maurer, U., Smailagic, A., Siewiorek, D.P., Deisher, M.: Activity recognition and monitoring using multiple sensors on different body positions. In: Proceedings of International Workshop on Wearable and Implantable Body Sensor Networks. Cambridge (2006)

25.Parkka, J., Ermes, M., Korpipaa, P. et al.: Activity classification using realistic data from wearable sensors. IEEE Trans. Inf. Technol. Biomed., 10(1), 119-128 (2006)

26.Besson, P., Richiardi, J., Bourdin, C., Bringoux, L., Mestre, D.R., Vercher, J.L.: Bayesian networks and information theory for audio-visual perception modeling. Biol. Cybern., 103(3), 213-226 (2010)

27.Rybarczyk, Y., Zalakeviciute, R.: Machine learning approach to forecasting urban pollution: a case study of Quito, Ecuador. In: Proceedings of IEEE Ecuador Technical Chapters Meeting. Guayaquil (2016)

28.Salvador, S., Chan, P.: Toward accurate dynamic time warping in linear time and space. Intell. Data Anal., 11(5), 561-580 (2007)

29.John, G.H., Langley, P.: Estimating continuous distributions in Bayesian classifiers. In: 11th Conference on Uncertainty in Artificial Intelligence. San Mateo (1995)

30.Kohavi, R., John, G.H.: Wrappers for feature subset selection. Artificial Intelligence, 97, 273-324 (1997) 